\title{
Predefined pattern detection in large time series
}

\author{
Shengfa Miao a , b , *, Ugo Vespier ${ }^{b}$, Ricardo Cachucho ${ }^{b}$, Marvin Meeng ${ }^{b}$, \\ Arno Knobbe ${ }^{b}$ \\ a Lanzhou University, Lanzhou, China \\ ${ }^{\mathrm{b}}$ Liacs, Leiden University, The Netherlands
}

\section{A R T I C L E I N F O}

\section{Article history:}

Received 1 April 2014

Revised 5 April 2015

Accepted 11 April 2015

Available online 17 April 2015

\section{Keywords:}

Pattern detection

Landmark

Trust region

MDL

Predefined pattern

Time series representation

\begin{abstract}
A B S T R A C T
Predefined pattern detection from time series is an interesting and challenging task. In order to reduce its computational cost and increase effectiveness, a number of time series representation methods and similarity measures have been proposed. Most of the existing methods focus on full sequence matching, that is, sequences with clearly defined beginnings and endings, where all data points contribute to the match. These methods, however, do not account for temporal and magnitude deformations in the data and result to be ineffective on several real-world scenarios where noise and external phenomena introduce diversity in the class of patterns to be matched. In this paper, we present a novel pattern detection method, which is based on the notions of templates, landmarks, constraints and trust regions. We employ the Minimum Description Length (MDL) principle for time series preprocessing step, which helps to preserve all the prominent features and prevents the template from overfitting. Templates are provided by common users or domain experts, and represent interesting patterns we want to detect from time series. Instead of utilising templates to match all the potential subsequences in the time series, we translate the time series and templates into landmark sequences, and detect patterns from landmark sequence of the time series. Through defining constraints within the template landmark sequence, we effectively extract all the landmark subsequences from the time series landmark sequence, and obtain a number of landmark segments (time series subsequences or instances). We model each landmark segment through scaling the template in both temporal and magnitude dimensions. To suppress the influence of noise, we introduce the concept of trust region, which not only helps to achieve an improved instance model, but also helps to catch the accurate boundaries of instances of the given template. Based on the similarities derived from instance models, we introduce the probability density function to calculate a similarity threshold. The threshold can be used to judge if a landmark segment is a true instance of the given template or not. To evaluate the effectiveness and efficiency of the proposed method, we apply it to two real-world datasets. The results show that our method is capable of detecting patterns of temporal and magnitude deformations with competitive performance.
\end{abstract}

(c) 2015 Elsevier B.V. All rights reserved.

\footnotetext{
* Corresponding author at: Liacs, Leiden University, The Netherlands.

E-mail addresses: miaoshengfa@hotmail.com (S. Miao), u.vespier@liacs.leidenuniv.nl (U. Vespier), r.cachucho@liacs.leidenuniv.nl (R. Cachucho), m.meeng@liacs.leidenuniv.nl (M. Meeng), a.j.knobbe@liacs.leidenuniv.nl (A. Knobbe).
} 


\section{Introduction}

This paper focuses on the problem of detecting instances of predefined patterns from time series [15,56]. While most pattern detection algorithms in time series deal with discovering previously unknown, frequently recurring regularities in the data, here we assume that one or more example sequences (the templates) are provided by a domain expert, and instances of these need to be identified in the actual data. During this detection, one needs to allow for a certain degree of difference between the template and the instances, for example because the instance is somewhat longer or shorter in duration, the magnitude of the signal is different, or parts of the signal are either stretched or compressed in time (so-called warps).

Li Wei et al. [56] mention a number of use-cases that motivate the predefined pattern detection problem. For example, in ECG monitoring, a cardiologist may observe some interesting pattern that he or she wants to annotate, and flag any future occurrences, to be investigated by the cardiologist or fellow experts. Alternatively, in insect pest control, one would like to observe specific cases of harmful insects, as identified by specific patterns of audio signal (wing beats). In our application to infrastructure monitoring, the predefined pattern detection problem is relevant for specifying and detecting known disturbances in the data, that can then be removed from the signal, or accounted for in subsequent modelling steps. For example, when monitoring the structural health of a bridge, the measured signal is dominated by recurring and understandable peaks due to vehicles crossing the bridge and traffic jams. One can imagine an expert providing a template for each of these phenomena, after which all instances should be identified, regardless of the speed and weight of the vehicles (influencing the width and height of the hump in the signal), or the duration of the traffic jam.

When matching a predefined phenomenon (a template $[40,41,48]$ ) with the time series under investigation, it is not always required to involve every individual measurement in the selected interval and in the template. In fact, when a certain level of fuzzy matching is required, it makes sense to somehow simplify the signal, or extract some key features that are characteristic for the sequence in question. This condensed representation can then be used to compare the time series with the template, both effectively (the matching is only based on the characteristic aspects) and efficiently (no computation is wasted on insignificant details). Specifically when large time series with high sampling rates are concerned, and the matching is nontrivial due to warps, efficient representation methods can be helpful. A considerable number of such methods have been proposed in the past, including Symbolic Aggregate approXimation (SAX) [29], bit-level approximation [7], and Piecewise Aggregate Approximation (PAA) [25]. ${ }^{1}$ In this paper specifically, we focus on the representation of time series by means of landmarks [43] (also referred to as key-points [8], break-points [47] and change-points [37]), which can be thought of as those points in the time series that are obviously remarkable (peaks, valleys, inflection points, ...). Rather than matching every detail of the data and the template, only the landmarks will be matched, and subsequent landmarks will be checked for their relationship to one another.

We match the given template to the actual data in three steps. The first step involves transforming the time series into a sequence of landmarks, which preserves all the prominent features. The second step is landmark subsequence selection, which is based on constraints over the landmarks occurring in the templates. The third step is instance model construction, which introduces a trust region to model the time series segments corresponding to the selected landmark subsequence. Unlike most of the representation and similarity methods, which are designed mainly for full sequence matching [15], our proposed approach is capable of processing both full sequence and subsequence matching of various length, while being less sensitive to noise, and being able to handle deformations in both magnitude and temporal dimensions.

One of the challenges when extracting landmarks from actual data is the noise and high-frequency vibrations that are included. An obvious step to get rid of such distractions and to produce a set of meaningful landmarks is to convolve the signal with a smoothing kernel. The question now becomes what level of smoothing is appropriate for the template in question. Too much smoothing may cause one to miss characteristic landmarks in the data, and too little smoothing will cause an abundance of landmarks at every little disturbance in the data. We propose an MDL-based solution to this challenge, that picks the correct smoothing level. Minimum Description Length (MDL) $[20,49,51]$ is an information-theoretic model-selection framework that selects the best model according to its ability to compress the given data.

The contributions of this paper are summarised as follows:

- It provides a general definition of a template for time series.

- It proposes the use of landmarks: a triple involving temporal, magnitude and type information.

- It takes the relationship between landmarks within a landmark sequence as constraints for landmark subsequence selection.

- It introduces the concept of a trust region from the image processing domain [32] to time series to build a reliable instance model.

- It employs MDL [20,49,51] for selection of the right smoothing level for landmark extraction.

The rest of this paper is organised as follows. Section 2 gives the definitions of template and landmark, and specifies the task of predefined pattern detection. Section 3 introduces the concept of landmark constraints. The question of choosing the right smoothing level through MDL is discussed in Section 4. In Section 5, instance models are used to fit the template to

1 A comprehensive list of representation methods for time series is given in Section 7. 
candidate instances in the data. Section 6 evaluates the proposed method by applying it to two datasets. Section 7 gives a literature review of related work, followed by a conclusion in Section 8.

\section{Preliminaries}

In this section, we will review and define some notation used throughout this paper. We begin by reviewing the concept of time series and subsequences, then give precise definitions of templates and landmarks.

Definition 1. A time series $\mathbf{T}$ is an ordered sequence of $\mathrm{n}$ real values:

$$
\mathbf{T}=\left(t_{1}, t_{2}, \ldots, t_{n}\right), \quad t_{i} \in \mathbb{R}
$$

For pattern detection purpose, instead of considering the full time series, we pay more attention to some interesting intervals. Each interval is called a subsequence, which is formally defined as:

Definition 2. Given a time series $\mathbf{T}=\left(t_{1}, \ldots, t_{n}\right)$ of length $n$, a subsequence $\mathbf{S}$ of $\mathbf{T}$ is a series of length $1 \leq m \leq n$ consisting of contiguous time points from $\mathbf{T}$ :

$$
\mathbf{S}=\left(t_{i}, t_{i+1}, \ldots, t_{i+m-1}\right), \quad 1 \leq i \leq n-m+1
$$

In this work, we assume query patterns, referred to as templates, to be given or predefined by domain experts.

Definition 3. A template $\mathbf{H}$ is a time series of length $\mathrm{k}$ that can serve as a model:

$$
\mathbf{H}=\left(h_{1}, h_{2}, \ldots, h_{k}\right), \quad h_{i} \in \mathbb{R}
$$

A example is given in the Fig. 1. The template in the left picture of Fig. 1 stands for a piece of bird song [45], which helps us find recurring subsequences from the time series in the right picture of Fig. 1.

\subsection{Landmark extraction}

Although we expect the user to specify the predefined pattern in terms of a template, we will not be matching the template directly to subsequences of the given time series. Rather, we intend to extract important landmarks [43] from both the template and the time series, and use these to match more efficiently and effectively. A landmark is defined as follows:

Definition 4. Given a time series $\mathbf{T}=\left(t_{1}, t_{2}, \ldots, t_{n}\right)$, a landmark is a remarkable point in $\mathbf{T}$, specified by a triple $l$ :

$$
l=(\text { id,mt,type }), \quad i d \in \mathbb{N}, m t \in \mathbb{R}
$$

where id is the index of the landmark in the time series $\mathbf{T}, m t$ is the magnitude of the landmark, type is the peak type indicator, which can be a local extreme, an inflection point or some other notable characteristic of the time series at this point.

We need to employ a landmark extraction method to produce a sequence $\mathbf{L}$ of landmarks from a given time series. Such a method, generally identified as a function $E$, can be applied to obtain a sequence of remarkable points from a given time series, but equally, it can be used to produce such points from a template, as that is essentially a time series also.

Landmark extraction methods are typically application dependent. In general, local extrema of the time series are good landmark candidates. They are found by considering the zero-crossings of the first derivative of the series. These zero-crossings (roots) correspond to the extrema in the time series, which we assume to be of interest. The inflection points derived from the extrema in the first derivative time series can also be considered as landmark candidates. Such landmarks can be found by looking at the zero-crossings of the second derivative. For a discrete time series, it may happen that a root is between two successive points of the time series. In this situation, we refer to the point close to zero as the zero-crossing point.

A landmark sequence preserves the main features of the time series, but significantly reduces its representation size. As shown in Fig. 2, the subsequence of length 900 can be compressed to a landmark sequence of only 16 elements $\left(l_{1}, l_{2}, \ldots, l_{16}\right)$.

Given a landmark subsequence $\mathbf{L}_{a}=\left(l_{1}, l_{2}, \ldots, l_{m^{\prime}}\right)$ and a time series $\mathbf{T}$, there is a subsequence in the time series corresponding to it. The subsequence is referred as a landmark segment $\mathbf{L S}$. The index of the first point of $\mathbf{L S}$ is derived from the index of $l_{1}$, and that of the end point of $\mathbf{L S}$ is derived from the index of $l_{m^{\prime}}$.

\subsection{Predefined pattern detection}

With the definitions of templates and landmarks now established, we can proceed by formally specifying the main task that we are concerned with in this paper, as follows: 

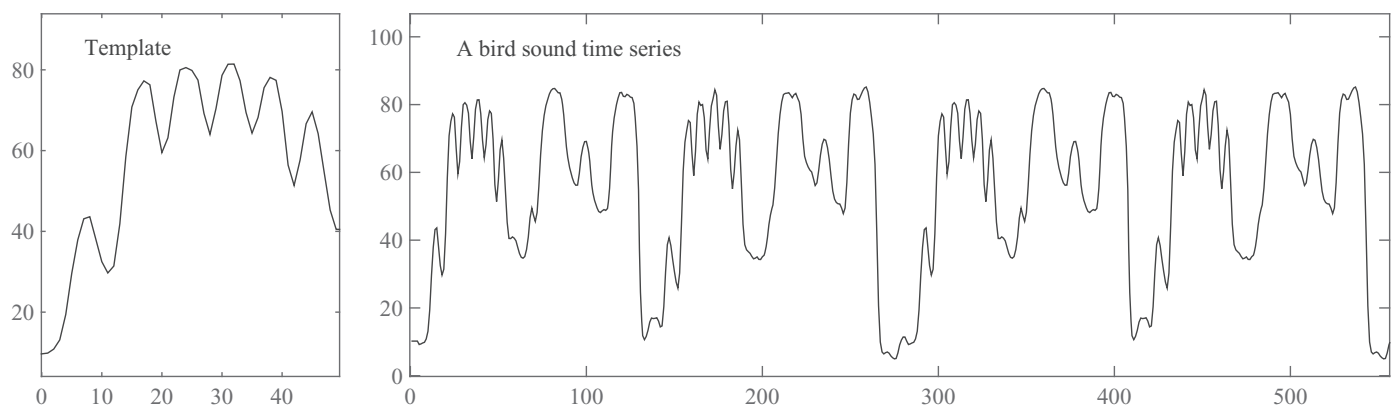

Fig. 1. An example of a template and time series. The curve in the left picture is a given template; the right picture illustrates a time series, in which subsequences similar to the template are expected.
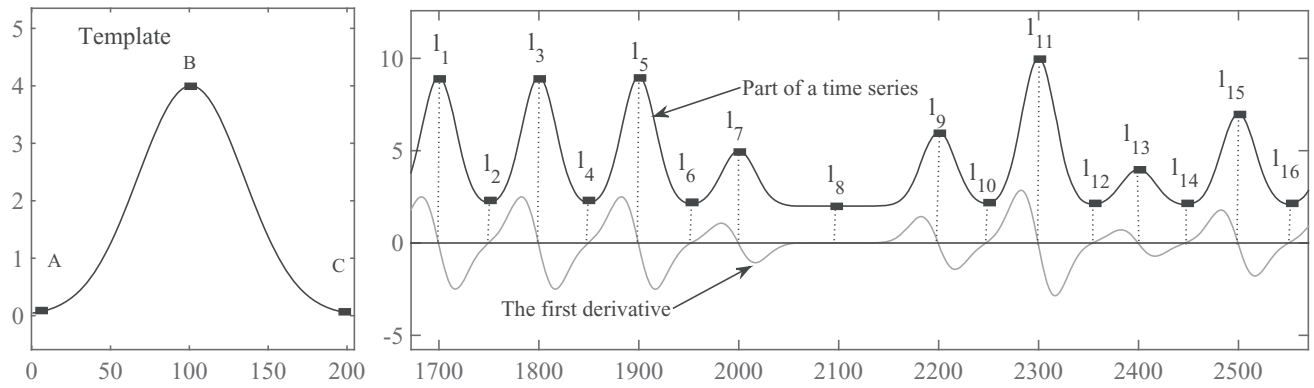

Fig. 2. The landmark sequence. The curve in the left picture is a template, marked with landmarks A, B and C; the black curve in the right picture is part of a time series, and the grey curve is its first derivative transform; the landmarks $l_{1}, l_{2}, \ldots, l_{16}$ are derived from the first derivative method.

Definition 5. The task of Predefined Pattern Detection takes as input a time series $\mathbf{T}$ of length $n$, a template $\mathbf{H}$ of length $k$ and a landmark extraction method $E_{\sigma}$, and produces a sequences of matches $\mathbf{M}=\left(L S_{1}, L S_{2}, \ldots, L S_{p}\right)$, where each $L S_{i}$ is a matched landmark segment in $\mathbf{T}, 0 \leq p \leq n$ is number of the matched subsequences.

There are two terms in the definition that should be addressed in more detail, one of which is the landmark extraction method $E_{\sigma}$. As mentioned, we are not matching the template to the time series directly, but rather extracting landmarks from both first, using $E_{\sigma}$. An important parameter in $E$ is $\sigma$, which helps to extract landmarks from the time series. If landmarks are defined as zero-crossings, $\sigma$ determines the level of smoothing applied to the raw time series. By smoothing, we prevent noise from playing a role in determining what constitutes a landmark. Of course, the level of noise (as opposed to the actual signal) depends on the application, so for the moment we assume this as simply a parameter of the task.

Another term is the sequence of matches $\mathbf{M}$. A time series subsequence is considered a matching landmark segment $L S_{i}$, if it can be conditionally transformed from the given template $\mathbf{H}$, with acceptable similarity. Given a template, we want to detect its instances from the time series $\mathbf{T}$. These instances are allowed to have a certain level of temporal or amplitude variation, but they should preserve all the prominent features of the template. The matches can be obtained with landmark constraints (in Section 3 ) and instance models (in Section 5).

In general, we assume the domain expert to provide additional information on the template in the form of constraints on the duration of the pattern as well as its magnitude. These will be expressed as lower and upper bounds on the duration of the pattern, as well as lower and upper bounds on the difference between the highest peak and the lowest valley. Clearly, without providing such constraints, one could stretch or compress the template to, say, a single data point or zero magnitude, losing all descriptive information in the template, while still matching many segments of the data.

\section{Landmark constraints}

In theory, for a given template landmark sequence of length $k^{\prime}$ and a time series landmark sequence of length $n^{\prime}$, there are $n^{\prime}-k^{\prime}+1$ candidate landmark subsequences. Compared with the subsequence candidates from the original time series, the number has already been reduced a lot. However, the candidate number can be further reduced by employing landmark constraints of template landmark sequence. In this section, we introduce landmark constraints to break the time series landmark sequence into a number of meaningful landmark subsequences.

For a given template, the landmarks in its landmark sequence signify more than just several data points obtained with landmark extraction methods. Constraints exist among the landmark sequence of the template. These constraints should be general enough to cover most potentially interesting patterns. 
For example, given the template of length 200 (marked with landmarks $A, B$ and $C$ ), shown as the left picture of Fig. 2, we are required to extract all the instances, whose lengths are between 80 and 350, from the time series, shown as the right picture of Fig. 2. The constraint set could be specified as follows:

- The length of landmark subsequences should be 3 .

- The first and last landmark of each subsequence should be valley points.

- The second landmark of each subsequence should be a peak point.

- The index difference between the last landmark and the first landmark should be between 80 and 350 .

The first three constraints are used to preserve the main features of the template, and the forth constraint is used to meet conditions provided by users or domain experts. With these constraints, the number of landmark subsequence candidates can be significantly reduced (for the given subsequence, the number is reduced from 14 to 7 , by $50 \%$ ). Each landmark subsequence corresponds to a landmark segment, so the number of landmark segments is also significantly reduced. We will introduce instance model to fit the selected landmark segments in the Section 5, which helps to further filter out false instances.

\section{Determining the smoothing level}

In practice, a time series, collected from a specific field, is composed of both meaningful events and noise. Sometimes, the meaningful events are even buried by the noise. In order to make landmark extraction methods work effectively, we need to smooth the time series. When smoothing a time series, there is clearly a trade-off at play. Smoothing too little will produce a time series that shows too many landmarks, and smoothing too much will remove most of local features, such that important landmarks may be overlooked (see Fig. 3). In this section, we tackle the challenge of setting an appropriate value for the smoothing scale $\sigma$ in $E_{\sigma}$.

Our solution to this challenge employs the Minimum Description Length principle [20]. The MDL principle states that, when choosing between several different candidate models of the data, the one that produces the cheapest encoding is the most desirable. In this context, the different candidate models are produced by different choices of the smoothing scale $\sigma$. In a nutshell, we consider a range of values for $\sigma$, applying landmark extraction method $E_{\sigma}$ to the smoothed time series. The idea of using MDL as a guiding principle to model various aspects of time series data has been introduced before in [22,51], but not with the specific intent of selecting an appropriate choice of $\sigma$.

\subsection{Minimum description length}

We concentrate on the two-part version of the MDL principle, which states that the best instance model IM to describe the time series $\mathbf{T}$ is the one that minimises the $\operatorname{sum} L(\mathbf{I M})+L(\mathbf{T} \mid \mathbf{I M})$, where

- $L(\mathbf{I M})$ is the cost, in bits, of the instance model derived from the given template.

- $L(\mathbf{T} \mid \mathbf{I M})$ is the length, in bits, of the description of the time series when encoded with the help of the instance model IM , that is the residual information not represented by IM .

A good, detailed model that catches most features of the target dataset leads to a low cost of $L$ (T) IM), but a good model also means a higher cost compared with a simple model. Therefore, a trade-off between model fit and its complexity is guaranteed by considering the size of the encoding. This property prevents the MDL method from overfitting.

When we calculate $L(\mathbf{I M})$ and $L(\mathbf{T} \mid \mathbf{I M})$, we assume that the values $t_{i}$ of the input time series $\mathbf{T}$ have been quantised to a finite number of symbols by employing the function defined below:

$$
Q\left(t_{i}\right)=\left\lfloor\left(t_{i}-\min (\mathbf{T})\right) /(\max (\mathbf{T})-\min (\mathbf{T})) \cdot N\right\rfloor-N / 2
$$

where $N$, assumed to be even, is the number of bins to use in the discretisation, while $\min (\mathbf{T})$ and $\max (\mathbf{T})$ are respectively the minimum and maximum value in $\mathbf{T}$. Throughout the rest of the paper, we assume $N=256$, in correspondence with similar work on MDL in time series [22,51]. One question that might arise is if such a quantisation removes meaningful information from the time series. In [22], the authors show that the effect of quantisation is rather modest on several time series from various domains.

\subsubsection{Encoding of the model}

We will first discuss the encoding of the instance model IM , which is derived from a given template. In the time series, the cost for encoding the instance model is composed of two parts: the index and the model parameters. The location of any instance is less than the total length of the time series $\mathbf{T}$, so it can be encoded with $\log _{2} n$ bits. Assuming there are $m$ parameters for each model ( $m$ is the cost of transformations), and each parameter can be modelled with $b$ bits, the total cost can be obtained by summing up these two parts: 

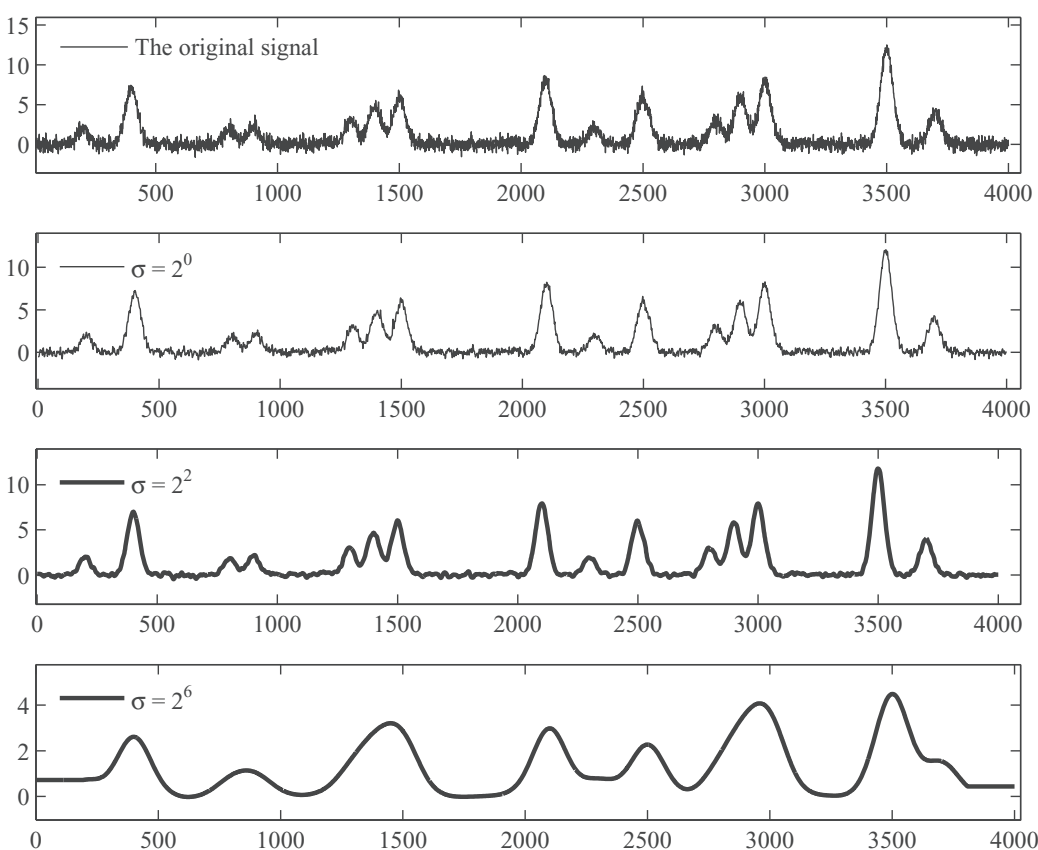

Fig. 3. Various scales of smoothing. The first picture shows the original time series without any preprocessing; the second picture shows the smoothed time series with a scale of $\sigma=2^{0}$, which still contains considerable noise; the third picture shows the smoothed time series with a scale of $\sigma=2^{2}$, which suppresses the noise and preserves the interesting patterns; the fourth pictures shows the smoothed time series with a scale of $\sigma=2^{6}$, which suppresses both the noise and the interesting features.

$$
L(\mathbf{I M})=k \cdot\left(\log _{2} n+m b\right)
$$

where $k$ is the number of instances in the time series that meet the landmark constraints.

\subsubsection{Encoding the data}

The second part of MDL, $L(\mathbf{T} \mid \mathbf{I M})$, represents the residual information after subtracting the instance model IM from the time series $\mathbf{T}$. To encode this part, we first need to introduce the notion of entropy.

Definition 6. The entropy of a time series $\mathbf{T}$, discretised according to a set of values $D$, is defined as below

$$
\operatorname{Entropy}(\mathbf{T})=-\sum_{v \in D} P\left(t_{i}=v\right) \log _{2} P\left(t_{i}=v\right)
$$

where $t_{i}$ stands for the $i_{\text {th }}$ element in the time series $\mathbf{T}, P \log _{2} P=0$ in the case of $P=0$, and $P\left(t_{i}=v\right)$ indicates the fraction of points in the time series which has value $v$.

Given the definition of entropy, we can define the description length of the second part of MDL as follows:

Definition 7. Given a time series $\mathbf{T}$ of length $n$, the description length of $L(\mathbf{T} \mid \mathbf{I M}$ ) (in bits) is given by

$$
L(\mathbf{T} \mid \mathbf{I M})=n \cdot \operatorname{Entropy}(\mathbf{T} \mid \mathbf{I} \mathbf{M})
$$

\subsection{Smoothing scale selection}

To assess the performance of a given smoothing scale $\sigma$, we first smooth the raw time series with the smoothing scale $\sigma$, and then transfer the smoothed time series Ts into landmark sequence with the landmark extraction method $E$. Under the landmark constraints derived from a given template, we can obtain a number of instances from the smoothed time series. The obtained instances work as instance models, and the residual, obtained by subtracting the values of the instance models from the related segments in the raw time series, works as the second part of MDL. We need two parameters (the indexes of the first and last data points of a landmark segment) to identify each instance model. Assuming there are $r$ interesting landmark segments (instances) under a smoothing scale $\sigma$, the instance model cost $L(\mathbf{I M})$ becomes:

$$
L(\mathbf{I M})=2 r \cdot \log _{2} n
$$

The second MDL part $L(\mathbf{T} \mid \mathbf{I M})$, according to Definition 7 is represented as: 


$$
L(\mathbf{T} \mid \mathbf{I M})=n \cdot \text { Entropy }\left(\mathbf{T}-\sum_{i=1}^{i=r} \mathbf{T} \mathbf{s}_{i}\right)
$$

where $\mathbf{T s}_{i}$ is the $i$ th landmark segment in the smoothed time series Ts .

For a given template and a set of smoothing scale candidates, we assume that the optimal smoothing scale is the one that leads to the minimal MDL score.

\section{Instance models}

Given a landmark segment LS of length $m$ and a template $\mathbf{H}$ of length $k$, we can simply model $\mathbf{L S}$ by scaling $\mathbf{H}$ in both temporal and magnitude dimensions. The temporal scale operation $X$-scale results in a new sequence $\mathbf{I M}_{X}$, whose length is the same as that of the landmark segment.

$$
\begin{aligned}
& \mathbf{I M}_{X}=X-\operatorname{scale}(\mathbf{H}, m, k), \quad k, m \in \mathbb{N} \\
& X-\text { ratio }=m / k
\end{aligned}
$$

If $X-$ ratio $>1$, the $X$-scale is an up-sampling operation, and if $X$-ratio $<1$, the $X$-scale is a down-sampling operation, otherwise, the $\mathbf{I M}_{X}$ is the same as the template $\mathbf{H}$.

We continue to process the obtained model $\mathbf{I M}_{X}$ with the magnitude scale operation $Y$ - scale, and obtain an instance model $\mathbf{I} \mathbf{M}_{Y}$, which can be taken as a primary approximation of the landmark segment $\mathbf{L S}$.

$$
\begin{aligned}
& \mathbf{I M}_{Y}=Y-\operatorname{scale}\left(\mathbf{H}, \mathbf{I} \mathbf{M}_{X}\right) \\
& Y-\operatorname{ratio}=(\max (\mathbf{L S})-\min (\mathbf{L S})) /(\max (\mathbf{H})-\min (\mathbf{H}))
\end{aligned}
$$

where $\mathbf{I} \mathbf{M}_{Y}$ is obtained by first scaling $\mathbf{I M}_{X}$ with a ratio $Y$ - ratio, resulting in a temporary model $\mathbf{I} \mathbf{M}_{X Y}$, then shifting along magnitude dimension by $\min (\mathbf{L S})-\min \left(\mathbf{I M}_{X Y}\right)$.

Based on the transformations mentioned above, we can define the instance model as:

Definition 8. Given a landmark segment $\mathbf{L S}$ of length $m$, and a template $\mathbf{H}$ of length $k$, the instance model $\mathbf{I M}$ of $\mathbf{L S}$ is a sequence derived from transformations of the template $\mathbf{H}$ :

$$
\mathbf{I M}=\operatorname{Trans} f(\mathbf{L S}, \mathbf{H})
$$

where Trans $f$ are the transformations defined above ( $X$ - scale and $Y$ - scale ).

The advantage of obtaining instance models with the transformations mentioned above, is that they are straightforward and work well with regular landmark segments. However, when warps or distortions exist in landmark segments, the transformations will become insufficient. To illustrate the problem, we present a template, shown as the curve in the top left picture of Fig. 4 , and use it to model three landmark segments: the curve in the top middle picture of Fig. 4 is the first landmark segments, which is a single peak; the curve in the top right picture of Fig. 4 is the second and the third landmark segments, which is composed of two overlapping peaks. The dotted curve IM1 in the bottom left picture of Fig. 4 is an instance model obtained by simply transforming the given template. The instance model indicates that the left boundary of the first landmark segment is incorrect, which is caused by the false landmark A1. The dotted curves IM2 and IM3 in the bottom right picture of Fig. 4 are two instances models, which are also obtained by simply transforming the given template. The boundaries of the landmark segments are correctly caught, but their instance models are still incorrect. This is caused by the complex (overlapping) nature of the time series. To overcome all these limitations, we introduce the notion of trust region.

\subsection{Trust region}

By assuming part of the landmarks within a landmark subsequence are reliable, in its landmark segment, we can define the corresponding segment between these landmarks as the trust region, shown as the thick regions in the top middle and right pictures of Fig. 4. Trust region is a concept borrowed from the field of image processing. We introduce the concept into time series (or to be more precise, into the instance model), and define it as:

Definition 9. Given a landmark segment $\mathbf{L S}$ of length $m$, and its landmark sequence $\mathbf{L}=\left(l_{1}, l_{2}, \ldots, l_{m^{\prime}}\right)$ of length $m^{\prime}$, if the segment between landmarks $l_{i}$ and $l_{j}\left(1 \leq i<j \leq m^{\prime}\right)$ is influenced less by noise, then the trust region of LS is defined as:

$$
\mathbf{L S}_{\text {trust }}=\left(t_{c 1}, \ldots, t_{d 1}\right), \quad 1 \leq c 1<d 1 \leq m
$$

where $c 1$ is the index of landmark $l_{i}$ in the landmark segment $\mathbf{L S}$, and $d 1$ corresponds to the index of landmark $l_{j}$. The dashed curves in the bottom left and right pictures of Fig. 4 are instance models obtained with the trust regions given in the top middle and right pictures, which indicate that the trust regions help to achieve improved instance models and updated landmark segments. The detailed procedure is illustrated in Algorithm 1. 

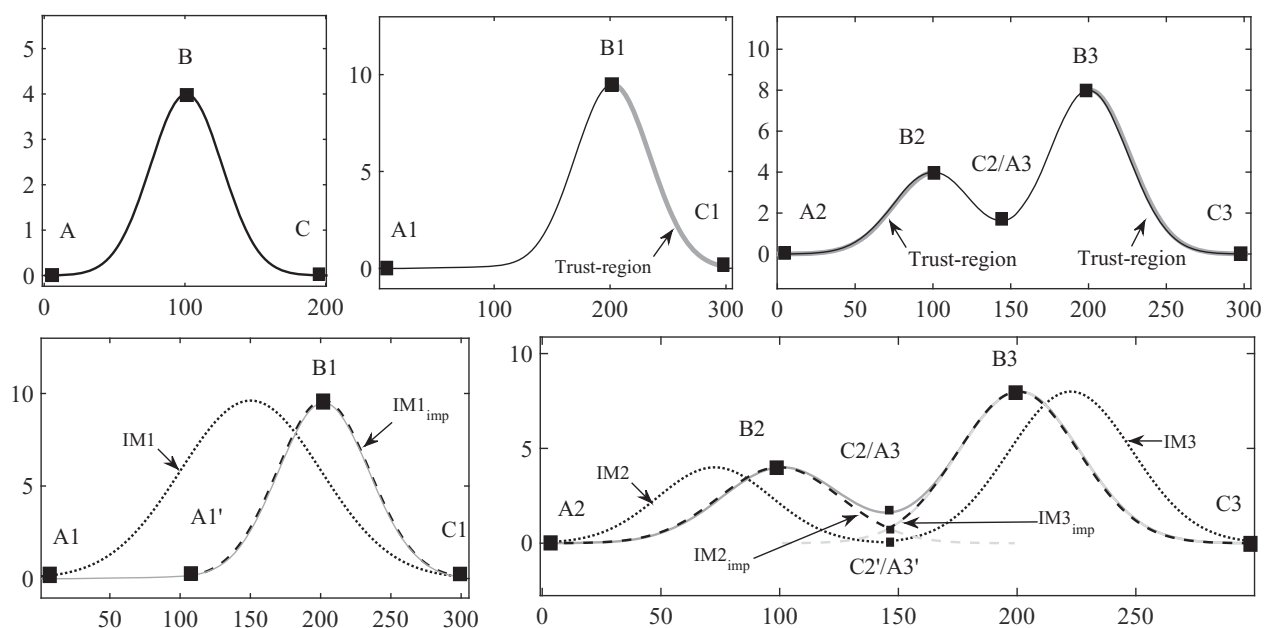

Fig. 4. The instance model. The curve in the top left picture is the given template, which is marked with landmarks $A$, $B$ and $C$; the segment between the landmarks B1 and C1 in the top middle picture is chosen as the trust region; in the top right picture, the segment between the landmarks A2 and B2 is the trust region of the first landmark segment, and that between the landmarks B3 and C3 is the trust region of the second landmark segment; the dotted curves in the bottom left and right pictures are instance models simply obtained with transformations of the given template; the dashed curves in the bottom left and right pictures are improved instance models based on the trust regions in the top middle and right pictures.

Algorithm 1. The instance model.

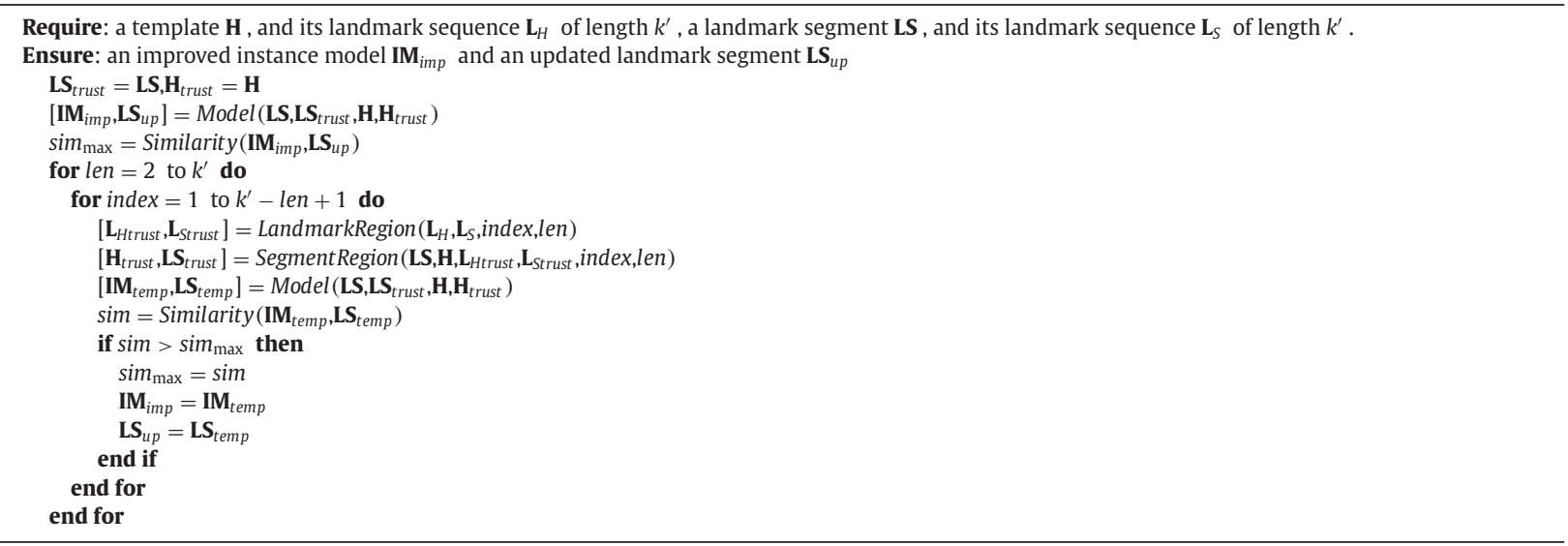

The inputs of the algorithm are: a template $\mathbf{H}$ of length $k$, a landmark segment $\mathbf{L S}$ of length $m$, and their landmark sequences $\left(\mathbf{L}_{H}\right.$ and $\mathbf{L}_{S}$, respectively, both of length $k^{\prime}$ ). The outputs of the algorithm are an improved instance model and an updated landmark segment.

Given a landmark sequence, we assume that the subsequence between the $i$ th $\left(1 \leq i \leq k^{\prime}\right)$ and the $j$ th $(1 \leq$ $j \leq k^{\prime}$ ) landmarks is reliable, which is referred as the trust landmark subsequence, and can be obtained with the LandmarkRegion $\left(\mathbf{L}_{H}, \mathbf{L}_{S}\right.$,index,len) operation. In the operation, index is equal to $i$, and len $($ len $=j-i+1)$ is the length of the trust landmark subsequence $\mathbf{L}_{H \text { trust }}$ of $\mathbf{L}_{H}$ or the trust landmark subsequence $\mathbf{L}_{\text {Strust }}$ of $\mathbf{L}_{S}$ ( $\mathbf{L}_{\text {Htrust }}$ and $\mathbf{L}_{\text {Strust }}$ are of the same length).

Assuming the corresponding indexes of the first landmark $l_{i}$ and the last landmark $l_{j}$ are $a 1$ and $b 1$ for $\mathbf{L}_{H t r u s t}$, and are $c 1$ and $d 1$ for $\mathbf{L}_{\text {Strust }}$. The trust region $\mathbf{H}_{\text {trust }}$ is the subsequence of $\mathbf{H}$, indexed by $a 1$ and $b 1$, and the trust region $\mathbf{L} \mathbf{S}_{\text {trust }}$ is the subsequence of $\mathbf{L S}$, indexed by $c 1$ and $d 1$. These trust regions can be obtained with the SegmentRegion $\left(\mathbf{L S}, \mathbf{H}, \mathbf{L}_{H t r u s t}, \mathbf{L}_{S t r u s t}\right.$, index,len $)$ operation. 
Based on the trust regions $\mathbf{H}_{\text {trust }}$ and $\mathbf{L} \mathbf{S}_{\text {trust }}$, the associated instance model $\mathbf{I M}_{\text {temp }}$ and the updated landmark segment $\mathbf{L} \mathbf{S}_{\text {temp }}$ can be obtained with the $\operatorname{Model}\left(\mathbf{L S}, \mathbf{L S}\right.$ trust $\left., \mathbf{H}, \mathbf{H}_{\text {trust }}\right)$ operation. The procedure of this operation is illustrated as follows:

- Temporal scaling: Based on the candidate trust regions $\mathbf{L S}_{\text {trust }}$ and $\mathbf{H}_{\text {trust }}$, we can obtain a new sequence IM $\mathbf{M}_{X}$ with the $X$ - scale operation:

$$
\mathbf{I M}_{X}=X-\operatorname{scale}(\mathbf{H}, d 1-c 1+1, b 1-a 1+1)
$$

The indexes of the $i$ th and the $j$ th landmarks in $\mathbf{I M}_{X}$ are $a^{\prime}$ and $b^{\prime}$. The length of $\mathbf{I} \mathbf{M}_{X}$ is $m 1$, which equals $\lfloor k$. (b1 $-a 1+$ $1) /(d 1-c 1+1)\rfloor$.

- Amplitude scaling: Following the same $Y$ - scale operation procedure as mentioned above, we obtain an instance model IM $\mathbf{M}_{Y}$ :

$$
\mathbf{I M}_{Y}=Y-\operatorname{scale}\left(\mathbf{H}, \mathbf{I M}_{X}\right)
$$

- Aligning and pruning: We should note that the lengths of $\mathbf{I M}_{Y}$ and $\mathbf{L S}$ may be different. To make them the same, we need to align and prune them. Assuming the indexes of the first and the last landmarks of $\mathbf{L S}$ are $c^{\prime}$ and $d^{\prime}$, and those of IM $\mathbf{M}_{Y}$ are 1 and $m 1$, the pre-trust region (the region before the trust region) lengths of $\mathbf{I M}_{Y}$ and $\mathbf{L S}$ are $\mathbf{L} \mathbf{S}_{\text {preL }}=c 1-c^{\prime}$ and $\mathbf{I} \mathbf{M}_{\text {preL }}=a^{\prime}-1$ respectively, and the post-trust region (the region after the trust region) lengths of $\mathbf{I M}_{Y}$ and $\mathbf{L S}$ are $\mathbf{L} \mathbf{S}_{p o s t L}=d^{\prime}-d 1$ and $\mathbf{I M}_{\text {postL }}=m 1-b^{\prime}$ respectively.

If $\mathbf{L S}_{\text {preL }}>\mathbf{I} \mathbf{M}_{\text {preL }}$ or $\mathbf{L} \boldsymbol{S}_{\text {post } L}>\mathbf{I} \mathbf{M}_{\text {postL }}$, we need to prune the landmark segment $\mathbf{L S}$, otherwise, fix the instance model IM $\mathbf{M}_{Y}$. The pruned landmark segment is $\mathbf{L} \mathbf{S}_{t e m p}$, and the fixed instance model is $\mathbf{I} \mathbf{M}_{t e m p}$.

Finally, the fit of each candidate instance model is evaluated with the Similarity() operation, which can be any of the choices as outlined in Section 7, most typically a similarity function based on the Euclidean Distance or Pearson's correlation.

\subsection{Model evaluation}

To judge if a landmark segment is an true instance of the template, we need to set a threshold on the similarity of the landmark segment and its instance model. We calculate the similarity threshold based on the probability density function (PDF) [36]. Assuming there are $j$ landmark segments that meet the landmark constraints, we can obtain a similarity sequence $\mathbf{S I M}=\left(\operatorname{sim}_{1}, \operatorname{sim}_{2}, \ldots, \operatorname{sim}_{j}\right)$, in which $\operatorname{sim}_{i}(1 \leq i \leq j)$ is the similarity between the $i$ th landmark segment and its instance model. We employ the kernel smoothed probability density function to detect the density distribution of SIM . The similarities of true instances are relatively high, and are assumed to produce a different density distribution from those of false instances. The changing point between the highest similarity density distribution and a lower similarity density distribution is taken as the similarity threshold. More formally, we determine the changing point by finding the first local minimum left of the highest peak in the PDF. Concrete examples are illustrated in Section 6. If a similarity is below this threshold, then the corresponding landmark segment will be taken as a false instance. Otherwise, we treat the landmark segment as a true instance.

\subsection{Complexity analysis}

Given a smoothing scale candidate set of length $s$, we need to computer $s$ convolutions to detect the right one. This can be done efficiently using the Fast Fourier Transform in $\mathcal{O}\left(s n \log _{2} n\right)$ time. For each smoothing scale, we then need to calculate the MDL score. This takes $\mathcal{O}(s n)$ time. The computation of the landmark detection can be done with a linear scan and thus has $\mathcal{O}(n)$ complexity. The transforms in instance models are linear operations, and both the number of landmark segments and the number of trust regions are relative small, so the complexity of this task also has $\mathcal{O}(n)$ complexity. Overall, our method has a complexity equal to the sum of these three complexities, which is $\mathcal{O}\left(\operatorname{sn} \log _{2} n\right)$ time.

\section{Experiments}

To show the effectiveness of the proposed method, we apply it to two real-life datasets (traffic and ECG signals). Based on a given template and prior knowledge, we begin with setting landmark constraints, then choose the right smoothing scale based on MDL. From the smoothed dataset, we can obtain a number of landmark segments. We model these segments with the given template, and obtain a number of instance models. The qualities of instance models are evaluated with similarities. In this paper, we choose correlation as the similarity method, and set a similarity threshold with PDF. If the similarity of an instance model is above the threshold, then the corresponding landmark segments will be taken as a true instance of the given template. 


\subsection{Traffic dataset}

The traffic dataset is collected from a highway bridge within the InfraWatch project [27,36,50]. The dataset is composed of 360,000 data points $(1 \mathrm{~h})$, sampled at $100 \mathrm{~Hz}$. There are 20 struck events and 173 car events within this dataset, as identified manually by inspecting the data and a video recording. Our task is to extract these traffic events from the datasets. Needless to say, the manual inspection is quite time consuming, and would be impractical for larger datasets. We address the task with the procedure mentioned in previous sections. From domain experts, we obtain a template of length 200, shown as the bottom left picture in Fig. 5. The sensor network is installed on one span of the bridge, whose length is $50 \mathrm{~m}$, and the local speed limit is $120 \mathrm{~km} / \mathrm{h}$, such that we can assume an event duration of at least $1.5 \mathrm{~s}$.

Based on the template, we take local extrema and inflection points as landmarks. The template is marked with five landmarks $(A, B, C, D$ and $E$ ). Based on the properties of these landmarks, we can further set constraints for landmark subsequence candidates:

- The length of landmark subsequences should be 5 .

- The first landmark and last landmark should be valley points.

- The second landmark and fourth landmark should be inflection points.

- The third landmark should be a peak point.

- The time between the first landmark and last landmark should be no less than $1.5 \mathrm{~s}$ (150 data points).

We employ the first derivative and second derivative methods to extract landmarks from the dataset. Derivative methods are sensitive to noise, so we need to smooth the original dataset before extracting landmarks from it, by means of a Gaussian Kernel. We employ MDL to select the proper smoothing scale $\sigma$ from a given set $\left\{\right.$ No smoothing, $\left.2^{0}, 2^{1}, 2^{2}, \ldots, 2^{9}\right\}$. In the top picture of Fig. 6 , the MDL-score as a function of the level of smoothing is indicated. As can be seen, the optimal value for $\sigma$ is $2^{4}$, which will be the smoothing level used for subsequent landmark extraction and matching with the template.

Based on the landmark constraints and the right smoothing scale, we obtain 245 landmark segments from the smoothed datasets. We continue to evaluate these segments with instance models. For each trust region, there is an instance model corresponding to it. Our template is marked with 5 landmarks, which can form 10 trust region candidates. In practice, the first landmark and the last landmark are sensitive to noise, so we just use the middle three landmarks, which generate 3 trust regions. We model each landmark segment based on these trust regions, and select the best-matching model. If the similarity between an obtained instance model and its landmark segment is higher than a given threshold, then the landmark segment will be taken as an instance of the given template. In this case, we choose Pearson's correlation as the similarity method, and explore the similarity threshold based on the probability density distribution of all similarities. As shown in the bottom picture of Fig. 6, the peak on the left hand side stands for the similarity distribution of true instances, and the index of the changing point (0.918) is taken as the similarity threshold.

Based on the similarity threshold, 221 out of 245 landmark segments are selected as traffic instances. Of these, 20 matches are truck events, corresponding to the 20 trucks identified manually. Of the remaining matches, 161 landmark segments are actual car events (out of the 173 cars), 40 landmark segments are false positives, where sections of noise are recognised as potential cars. The precision of the method is thus $81.9 \%$, and the recall is $93.8 \%$. Note that our recall is $100 \%$ for the trucks, which, due to their large size and impact on the strain on the bridge, are much easier to identify than cars. In Fig. 7 , we plot two features of the manually identified traffic events, demonstrating the case with which trucks can be distinguished
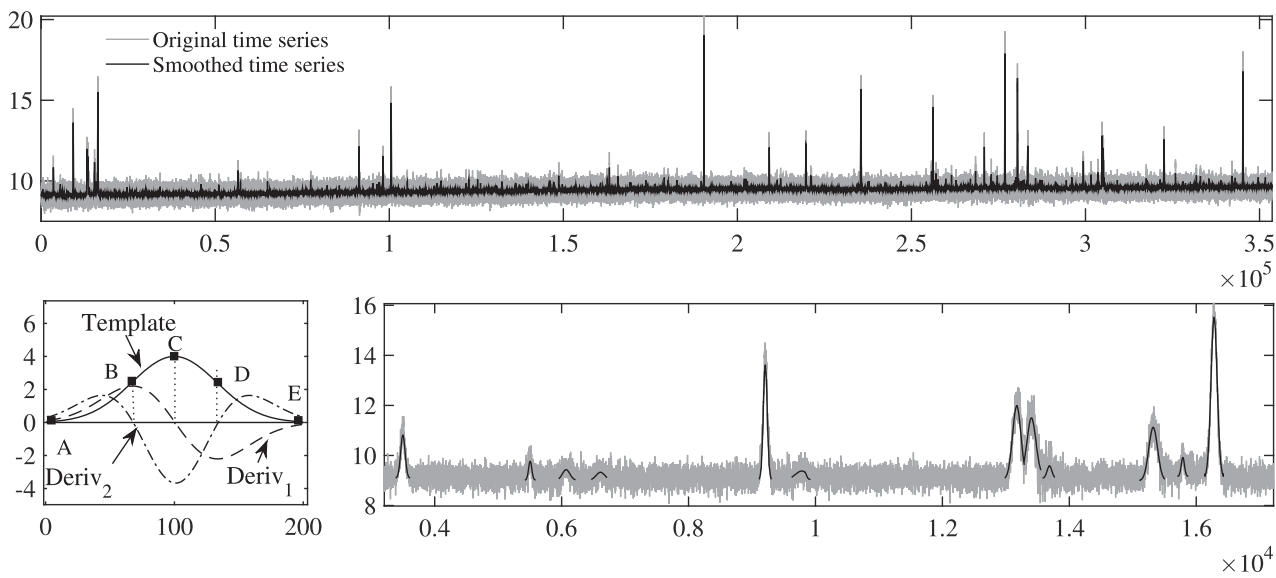

Fig. 5. Traffic event detection from a traffic dataset. The grey curve in the top picture is a raw dataset collected from a highway bridge; the black curve in the top picture is the smoothed dataset with the smoothing scale $\sigma=2^{4}$; the solid curve in the bottom left picture is the given template, marked with landmarks A, B, $\mathrm{C}, \mathrm{D}$ and $\mathrm{E}$; the dashed curve Deriv $v_{1}$ in the bottom left picture is the first derivative curve of the template; the dash-dotted curve Deriv $v_{2}$ in the bottom left picture is the second derivative curve of the template; the black peaks in the bottom right picture are instance models. 

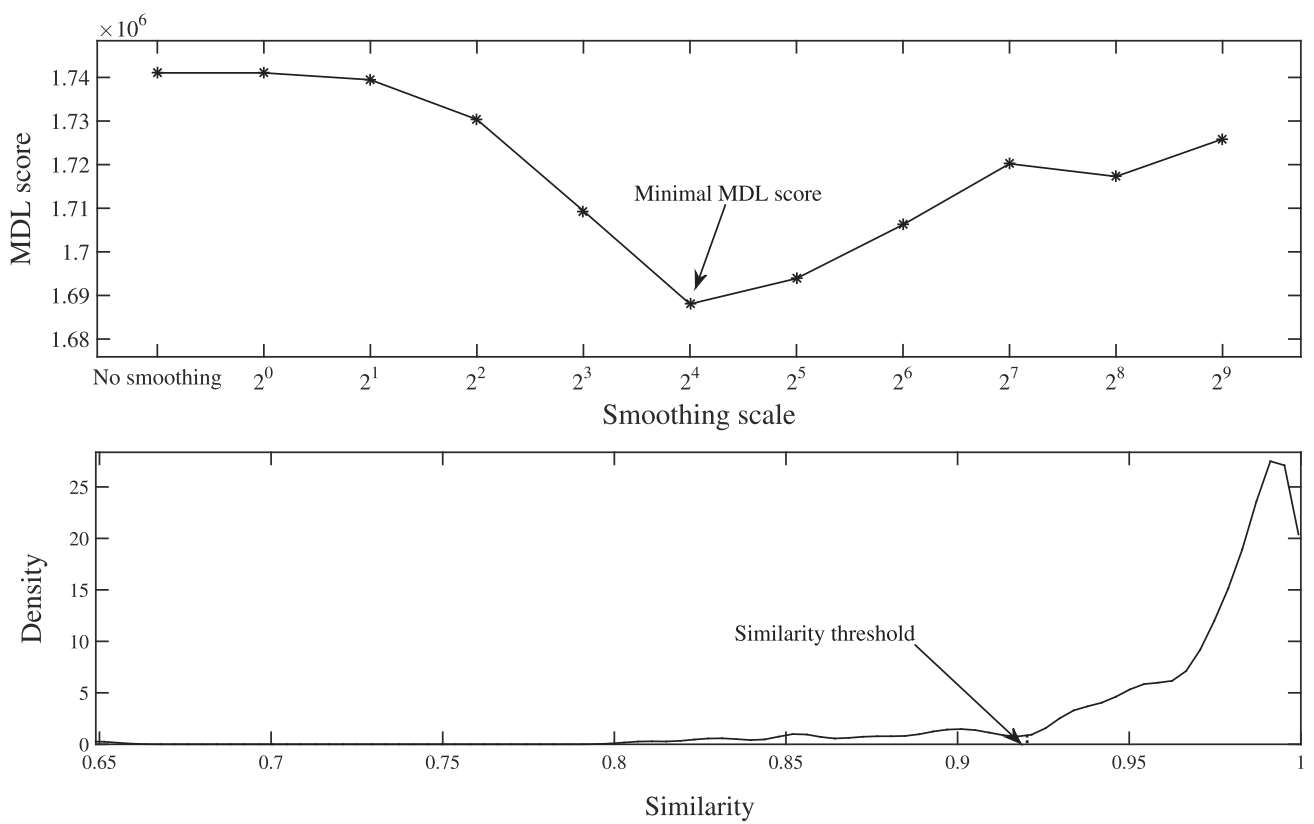

Fig. 6. The smoothing scale and similarity threshold of the traffic dataset. The top picture shows a scatter plot of the MDL score as a function of smoothing scale, in which the sixth smoothing scale $2^{4}$ is selected as the right smoothing scale; the bottom picture shows the probability density distribution of the similarities, the changing point 0.918 is selected as the similarity threshold.

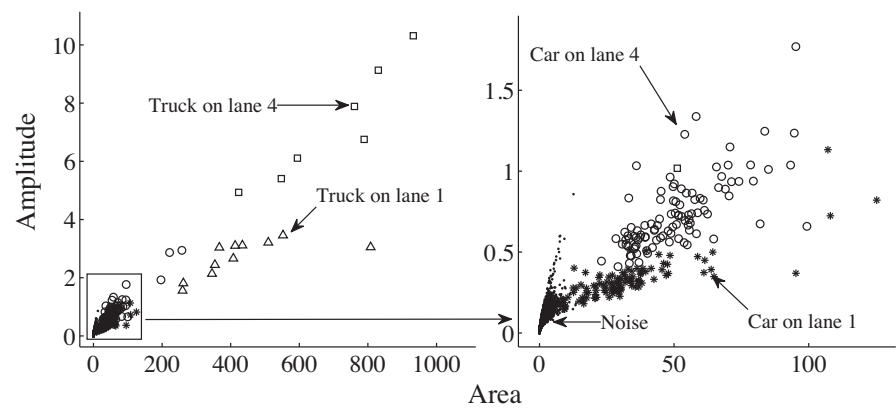

Fig. 7. Scatter plot of two features of traffic events. With two features (area and amplitude), traffic event candidates are classified into five groups (noise, car on lane 1, car on lane 4, truck on lane 1, truck on lane 4); the plot indicates that the bounds between noise and car events are blurred.

from cars, as well as traffic-free periods. In the detail in the right of this figure, we note how cars can sometimes be confused with traffic-free periods, due to the level of noise present in the data. Fig. 8 shows two car events in detail, which are clearly hidden by the serious level of noise in the data. Even after being smoothed, the segments are seriously distorted, and cannot be matched with the given template.

\subsection{ECG signal}

The electrocardiogram (ECG) signal is used to measure the electrical activity of the (human) heart [53]. A single heart beat is typically composed of 5 deflections, called the $\mathrm{P}, \mathrm{Q}, \mathrm{R}, \mathrm{S}$ and $\mathrm{T}$ wave, in which the $\mathrm{Q}, \mathrm{R}$ and $\mathrm{S}$ waves are often considered together as the QRS complex, because they are closely linked. Note that not every QRS complex contains all the three wave elements, and any combination of these waves can also be referred to as a QRS complex [1]. Accurately recognising the QRS complex and distinguishing them from the other noise sources such as P and T waves is a critical technology for many clinical instruments [2].

In this section, we choose an ECG dataset of 20,000 data points from [26], which contains 119 QRS complexes, and is collected at a frequency of $250 \mathrm{~Hz}$. The curve in the bottom left picture of Fig. 9 is a QRS template, which is composed of an R wave and an $\mathrm{S}$ wave. The template is marked with four landmarks $(A, B, C$, and $D)$. All the landmarks are local extrema, which can be extracted with the first-derivative method. The length of the template is 16 , and its amplitude (the amplitude difference between the landmark $B$ and the landmark $C$ ) is 0.449 . From domain experts, we learn that the QRS complex normally 


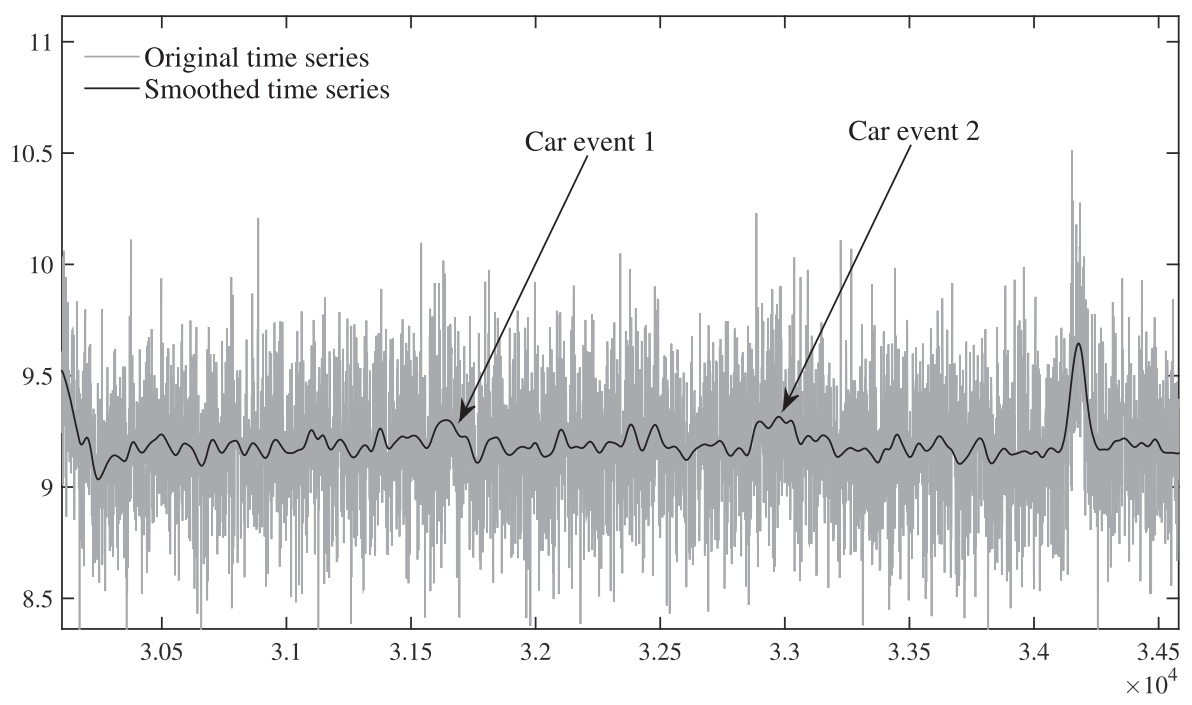

Fig. 8. Car events in the traffic dataset. This picture shows two car events with a lot of noise, such that it is very hard for a human to even recognise where the car events are.
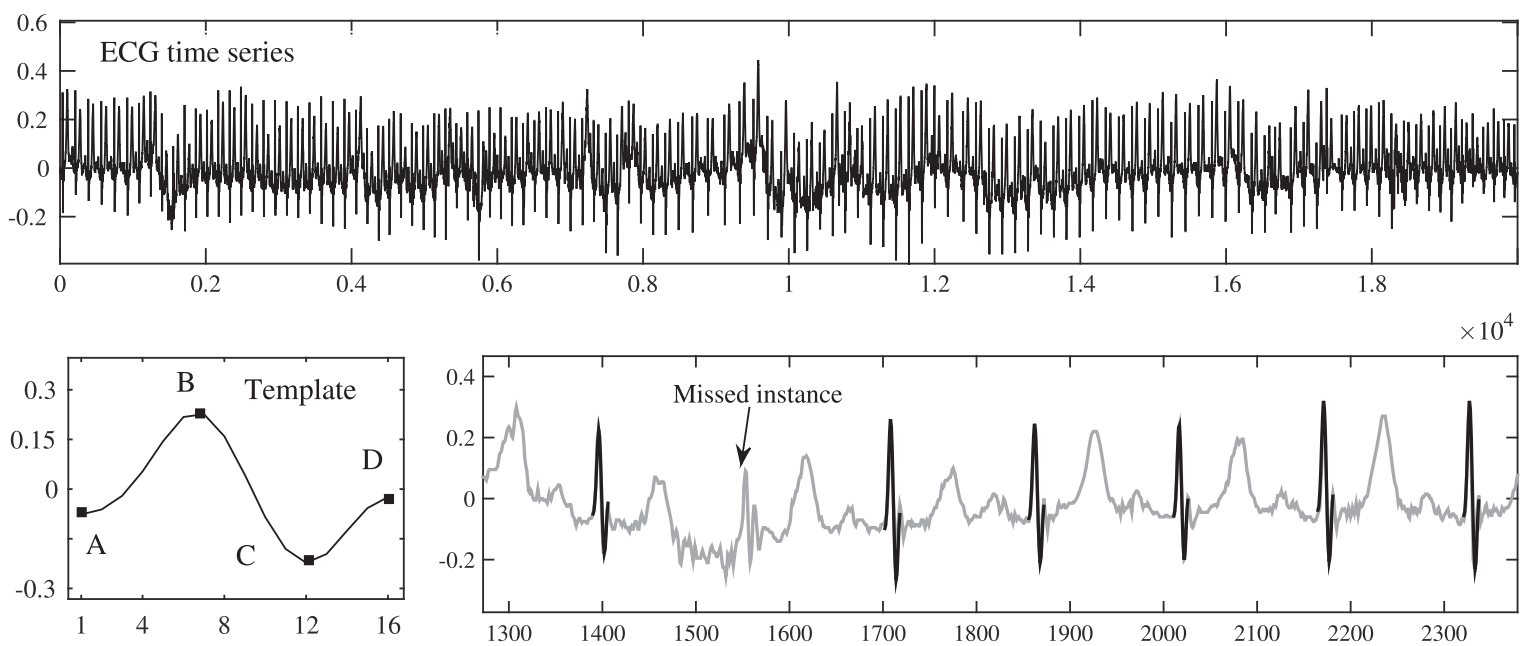

Fig. 9. The QRS complex detection from an ECG dataset. The grey curve in the top picture is an ECG dataset taken from a real patient; the curve in bottom left picture is a template of QRS complex, which is composed of an R wave and an S wave, and can be marked with 4 landmarks A, B, C and D; in the bottom right picture, black curves stand for matched instance models, and there is also an example of unmatched QRS complex.

lasts $0.06-0.10 \mathrm{~s}$, which is $15-25$ data points (at $250 \mathrm{~Hz}$ ). To be on the safe side, we assume the length of QRS instances ranges between 10 and 30 points. Being the most visually obvious part of ECG signals, the QRS complex features a high R wave and a low $S$ wave, marked as $B$ and $C$ in the template. The amplitude difference between $B$ and $C$ varies with QRS instances, but should also be within a reasonable range. In this experiment, we assume the amplitude range is between 0.2 and 0.8 . Based on this prior knowledge, the landmark constraints are set as:

- The length of each landmark subsequence should be 4 .

- The first and third landmarks should be valley points.

- The second and the fourth landmarks should be peak points.

- The magnitude of the second landmark should be the highest one in the landmark subsequence.

- The magnitude of the third landmark should be the lowest one in the landmark subsequence.

- The temporal difference between the last and the first landmark should be between 10 and 30 .

- The magnitude difference between the second and the third landmark should be between 0.2 and 0.8 .

Following the same smoothing scale set as the previous experiment, we calculate the MDL scores of each scale. As shown in the top picture of Fig. 10 , the original ECG dataset has the minimal MDL score, which indicates that the ECG dataset is best 

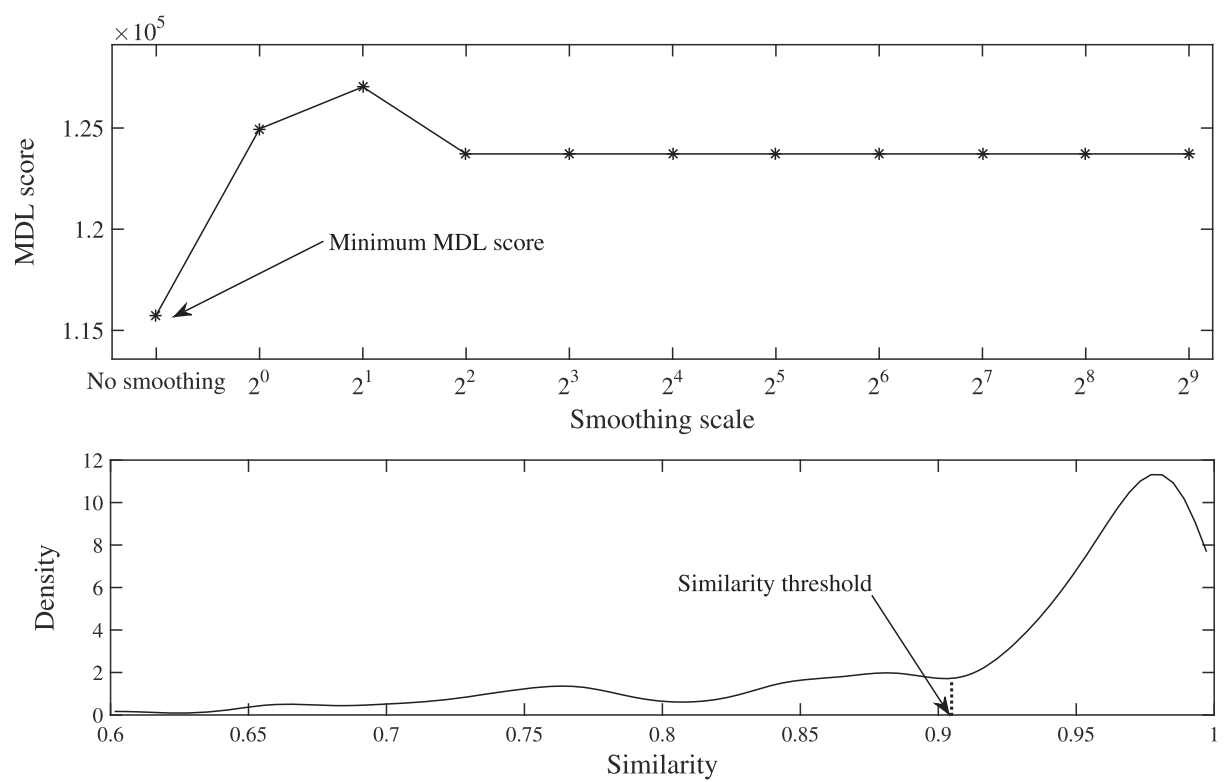

Fig. 10. The smoothing scale and similarity threshold for the ECG dataset. This top picture shows a scatter plot of the MDL score as a function of smoothing scale, and indicates that the ECG dataset need not be smoothed; the bottom picture shows the probability density distribution of the similarities. The changing point 0.901 is selected as the similarity threshold.

left unsmoothed. Based on landmark constraints set above, we manage to catch 142 landmark segments from the original ECG dataset. We continue to detect the similarity threshold with the probability density distribution of the similarities of selected segments. As shown in the bottom picture of Fig. 10, the index of the changing point (0.901) is selected as the similarity threshold. Based on the similarity threshold, 110 out of 142 landmark segments are selected as QRS instances. Of these, 108 are true QRS complexes and 2 are false QRS complexes. The precision of the instance model is thus $90.8 \%$, and the recall is $97.3 \%$.

\section{Related work}

In this paper, we have presented three concepts that have been extensively used in image matching fields: templates [5,31], landmarks [31,33] and trust regions [32].

Template matching can be used for face detection [23], duplicate document detection [10] and motion classification [38]. The concept of template has been introduced to time series to detect specific patterns or shapes [18,19,40,41,48]. Frank et al. [18] propose Geometric Template Matching (GeTeM) which uses time-delay embeddings for building models from segments of time series and compares the reconstructed dynamical systems in terms of their state space as well as their dynamics. In [19], a novel and flexible approach is proposed based on segmental semi-Markov models. In [40,41,48], meaningful templates are constructed with shape-based averaging algorithms, such as Prioritized Shape Averaging (PSA) [40] and Accurate Shape Averaging (ASA) [48]. Wei et al. propose the Atomic Wedgie method "that exploits the commonality among the predefined patterns to allow monitoring at higher bandwidths, while maintaining a guarantee of no false dismissals" [56]. Most of the proposed methods are mainly designed for full sequence matching, which are ineffective in detecting predefined patterns from time series.

Landmarks can be used to break time series into meaningful segments, which are also referred to as key-points [8], breakpoints [47] and change-points [37]. Perng et al. [43] propose a feature-based technique, which uses landmarks instead of the raw data for processing. A two-level representation [8] is proposed to recognise gestures, using both local and global features. In practice, the reliability of each landmark varies with its location. To the best of our knowledge, this has not been mentioned in the literature.

It has been pointed out by researchers that some unspecified portions of time series should be ignored $[3,45]$ to achieve a better matching result, which means some data points have nothing to do with predefined patterns, and should be filtered out. Ye and Keogh [57] propose a new time series primitive, time series shapelets, for time series classification. The shapelets are informally defined as the subsequences that are in some sense maximally representative of a class. This method is interpretable and accurate in classifying static time series [42], but is ineffective in handling real time time series. Inspired by these works, we introduce trust region into time series to obtain more reliable instance models.

A number of representation methods have been developed in the literature to reduce the dimensionality of time series, such as Discrete Fourier Transform (DFT) [17], Single Value Decomposition (SVD) [28], Discrete Wavelet Transform (DWT) [12]. There are also some researchers who employ symbolic representations, such as Symbolic Aggregate approximation 
(SAX) [29] and bit-level approximation [7]. Features extracted from time series carry summarised information of the time series [4,55], which can represent the original time series concisely [21], and are less sensitive to noise [39], so the feature extraction operation can also be used to reduce dimensionality (reduce the size of the data), such as Amplitude-Level Features (ALF) [6], Characteristic-based Clustering (CBC) [55]. Some representations are based on piecewise techniques, such as Piecewise Linear Approximation (PLA) [47], Piecewise Aggregate Approximation (PAA) [25], Adaptive Piecewise Constant Approximation (APCA) [11], Derivative Time Series Segment Approximation (DSA) [21] and Piecewise Vector Quantized Approximation (PVQA) [34,54]. Some representations aim to keep both local and global information about the original time series, such as Multi-resolution Vector Quantization (MVQ) approximation [35] and multi-resolution PAA (MPAA) [30].

Next to the representation methods, a number of similarity measures have been proposed [16], of which the Euclidean Distance (ED) [17,58] is the most common [4,15]. However, when shifting and temporal distortions exist in the given time series, the ED is proven to be ineffective [15]. To handle stretching and compression along the temporal dimension, Dynamic Time Warping (DTW) [9] was proposed, which achieves an optimal temporal alignment through detecting the shortest warping path in a distance matrix $[16,41,44,48]$. Finding the shortest warping path is a non-trivial problem, whose computation complexity can reach $O\left(n^{2}\right)$, where $n$ is the number of data points. To speed up the computation of DTW, some lower bounding constraints, like LB_Keogh [24,44] and the Ratanamahatana-Keogh Band [46], have been introduced to prune expensive computations, which can reduce the complexity to $O(n)$. There are also some other edit-based methods proposed to handle outliers and noise [16], such as Longest Common Subsequence (LCSS) [52], Edit Distance with Real Penalty (ERP) [13] and Edit Distance on Real sequence (EDR) [14]. However, most of the proposed methods focus mainly on temporal deformations [19], which are inadequate in dealing with shifting and scaling in the amplitude dimension [15]. Consequently, Spatial Assembling Distance (SpADe) [15] is proposed to handle shifting and scaling in both the temporal and amplitude dimensions.

\section{Conclusion}

Predefined pattern detection from time series is a quite challenging topic, because it is not only sensitive to noise, but also sensitive to temporal and magnitude deformations. A number of representation and similarity measure methods have been proposed to approximate interesting subsequences, but most of them are mainly designed for full sequence matching, and are ineffective when the disturbances mentioned above exist. We refer a predefined pattern as a template, and featured it with a sequence of landmarks (important points). Instead of comparing the template with subsequences of a given times series, we first transfer the time series into a landmark sequence, and compare the template landmark sequence with the time series landmark subsequences. The landmark constraints derived from the template and prior knowledge play a key role in finding matched landmark subsequences and landmark segments. We model the landmark segments with the template, and obtain a number of instance models. If the similarity between a landmark segment and its instance model is above a threshold, then the segment will be taken as an instance of the template. We introduce advanced methods, like MDL and PDF, to set important parameters, such as smoothing scale and similarity threshold. Most of the existing feature-based methods just focus on the quality of models, and pay little attention to the reliability of candidate patterns. Our instance model overcomes this problem by transferring the template in both temporal and magnitude dimensions according to trust regions.

\section{Acknowledgments}

This work and the InfraWatch project are funded by the Dutch funding agency (STW), under Project No. 10970.

\section{References}

[1] 2014. <http://en.wikipedia.org/wiki/QRS_complex>

[2] V.X. Afonso, ECG QRS detection, Biomedical Digital Signal Processing: C-language Examples and Laboratory Experiments for the IBM PC, 1993, pp. $236-264$.

[3] R. Agrawal, K. Lin, H. Sawhney, K. Shim, Fast similarity search in the presence of noise, scaling, and translation in time-series databases, in: Proceedings of International Conference on Very Large Databases, 1995, pp. 490-501.

[4] R.J. Alcock, Y. Manolopoulos, Time-series similarity queries employing a feature-based approach, in: Proceedings of Hellenic Conference on Informatics, 1999, pp. 27-29.

[5] Y. Amit, U. Grenander, M. Piccioni, Structural image restoration through deformable templates, J. Am. Stat. Assoc. 86 (1991) 376-387.

[6] J. Aßfalg, H.-P. Kriegel, P. Kröger, P. Kunath, A. Pryakhin, M. Renz, Similarity search in multimedia time series data using amplitude-level features, in: Proceedings of International Conference on Advances in Multimedia Modeling, 2008, pp. 123-133.

[7] A. Bagnall, C. Ratanamahatana, E. Keogh, S. Lonardi, G. Janacek, A bit level representation for time series data mining with shape based similarity, Data Min. Knowl. Discov. 13 (2006) 11-40.

[8] J.P. Bandera, R. Marfil, A. Bandera, J.A. Rodríguez, L. Molina-Tanco, F. Sandoval, Fast gesture recognition based on a two-level representation, Pattern Recogn. Lett. 30 (2009) 1181-1189.

[9] D.J. Berndt, J. Clifford, Using dynamic time warping to find patterns in time series, in: Proceedings of KDD Workshop, 1994, pp. 359-370.

[10] R. Caprari, Duplicate document detection by template matching, Image Vision Comput. 18 (8) (2000) 633-643.

[11] K. Chakrabarti, E. Keogh, S. Mehrotra, M. Pazzani, Locally adaptive dimensionality reduction for indexing large time series databases, ACM Trans. Database Syst. 27 (2) (2002) 188-228.

[12] K. Chan, A. Fu, Efficient time series matching by wavelets, in: Proceedings of International Conference on Data Engineering, 1999, pp. 126-133.

[13] L. Chen, R. Ng, On the marriage of Lp-norms and edit distance, in: Proceedings of International Conference on Very Large Data Bases, 2004, pp. 792-803. 
[14] L. Chen, M.T. Özsu, V. Oria, Robust and fast similarity search for moving object trajectories, in: Proceedings of International Conference on Management of Data, 2005, pp. 491-502.

[15] Y. Chen, M. Nascimento, B. Ooi, A. Tung, Spade: on shape-based pattern detection in streaming time series, in: Proceedings of International Conference on Data Engineering, 2007, pp. 786-795.

[16] P. Esling, C. Agon, Time-series data mining, ACM Comput. Surv. (CSUR) 45 (1) (2012) 12:1-12:34.

[17] C. Faloutsos, M. Ranganathan, Y. Manolopoulos, Fast subsequence matching in time-series databases, in: Proceedings of the International Conference on Management of Data, 1994, pp. 419-429.

[18] J. Frank, S. Mannor, J. Pineau, D. Precup, Time series analysis using geometric template matching, IEEE Trans. Pattern Anal. Machine Intell. 35 (3) (2013) $740-754$.

[19] X. Ge, P. Smyth, Deformable markov model templates for time-series pattern matching, in: Proceedings of International Conference on Knowledge Discovery and Data Mining, 2000, pp. 81-90.

[20] P.D. Grünwald, The Minimum Description Length Principle, The MIT Press, 2007.

[21] F. Gullo, G. Ponti, A. Tagarelli, S. Greco, A time series representation model for accurate and fast similarity detection, Pattern Recogn. 42 (2009) $2998-3014$.

[22] B. Hu, T. Rakthanmanon, Y. Hao, S. Evans, S. Lonardi, E. Keogh, Discovering the intrinsic cardinality and dimensionality of time series using MDL, in: Proceedings of International Conference on Data Mining, 2011, pp. 1086-1091.

[23] Z. Jin, Z. Lou, J. Yang, Q. Sun, Face detection using template matching and skin-color information, Neurocomputing 70 (4-6) (2007) 794-800.

[24] E. Keogh, Exact indexing of dynamic time warping, in: Proceedings of International Conference on Very Large Data Bases, 2002 , pp. 406-417.

[25] E. Keogh, K. Chakrabarti, M. Pazzani, S. Mehrotra, Dimensionality reduction for fast similarity search in large time series databases, Knowl. Inform. Syst. 3 (2001) 263-286.

[26] E. Keogh, Q. Zhu, B. Hu, Y. Hao, X. Xi, L. Wei, C.A. Ratanamahatana, The UCR time series classification/clustering page, 2011. < http://www.cs.ucr.edu/eamonn/ time_series_data/>.

[27] A. Knobbe, H. Blockeel, A. Koopman, T. Calders, B. Obladen, C. Bosma, H. Galenkamp, E. Koenders, J. Kok, Infrawatch: data management of large systems for monitoring infrastructural performance, in: Proceedings of Intelligent Data Analysis, 2010, pp. 91-102.

[28] F. Korn, H.V. Jagadish, C. Faloutsos, Efficiently supporting ad hoc queries in large datasets of time sequences, in: Proceedings of International Conference on Management of Data, 1997, pp. 289-300.

[29] J. Lin, E. Keogh, S. Lonardi, B. Chiu, A symbolic representation of time series, with implications for streaming algorithms, in: Proceedings of ACM SIGMOD Workshop on Research Issues in Data Mining and Knowledge Discovery, 2003, pp. 2-11.

[30] J. Lin, M. Vlachos, E. Keogh, D. Gunopulos, J. Liu, S. Yu, J. Le, A MPAA-based iterative clustering algorithm augmented by nearest neighbors search for timeseries data streams, in: Proceedings of Pacific-Asia Conference on Knowledge Discovery and Data Mining, 2005, pp. 333-342.

[31] R. Liu, Y. Lu, C. Gong, Y. Liu, Infrared point target detection with improved template matching, Infrared Phys. Technol. 55 (4) (2012) 380-387.

[32] T. Liu, H. Chen, Real-time tracking using trust-region methods, IEEE Trans. Pattern Anal. Machine Intell. 26 (3) (2004) $397-402$.

[33] M. Lüthi, C. Jud, T. Vetter, Using landmarks as a deformation prior for hybrid image registration, in: Proceedings of International Conference on Pattern Recognition, 2011, pp. 196-205.

[34] V. Megalooikonomou, G. Li, Q. Wang, A dimensionality reduction technique for efficient similarity analysis of time series databases, in: Proceedings of International Conference on Information and Knowledge Management, 2004, pp. 160-161.

[35] V. Megalooikonomou, Q. Wang, G. Li, C. Faloutsos, A multiresolution symbolic representation of time series, in: Proceedings of International Conference on Data Engineering, 2005, pp. 668-679.

[36] S. Miao, E. Koenders, A. Knobbe, Automatic baseline correction of strain gauge signals, Struct. Control Health Monit. 22 (2015) $36-49$.

[37] Y. Mohammad, T. Nishida, Constrained motif discovery in time series, New Gener. Comput. 27 (2009) 319-346.

[38] M. Müller, T. Röder, Motion templates for automatic classification and retrieval of motion capture data, in: Proceedings of ACM SIGGRAPH/Eurographics Symposium on Computer Animation, 2006, pp. 137-146.

[39] A. Nanopoulos, R. Alcock, Y. Manolopoulos, Feature-based classification of time-series data, Int. J. Comput. Res. 10 (2001) 49-61.

[40] V. Niennattrakul, C. Ratanamahatana, Shape averaging under time warping, in: Proceedings of International Conference on Electrical Engineering/Electronics, Computer, Telecommunications, and Information Technology, 2009, pp. 626-629.

[41] V. Niennattrakul, D. Srisai, C. Ratanamahatana, Shape-based template matching for time series data, Knowl.-Based Syst. 26 (2012) 1-8.

[42] T. Palpanas, M. Vlachos, E. Keogh, D. Gunopulos, W. Truppel, Online amnesic approximation of streaming time series, in: Proceedings of International Conference on Data Engineering, 2004, pp. 338-349.

[43] C.-S. Perng, H. Wang, S.R. Zhang, D.S. Parker, Landmarks: a new model for similarity-based pattern querying in time series databases, in: Proceedings of International Conference on Data Engineering, 2000, pp. 33-42.

[44] T. Rakthanmanon, B. Campana, A. Mueen, G. Batista, B. Westover, Q. Zhu, J. Zakaria, E. Keogh, Searching and mining trillions of time series subsequences under dynamic time warping, in: Proceedings of International Conference on Knowledge Discovery and Data Mining, 2012, pp. 262-270.

[45] T. Rakthanmanon, E. Keogh, S. Lonardi, S. Evans, Time series epenthesis: Clustering time series streams requires ignoring some data, in: Proceedings of International Conference on Data Mining, 2011, pp. 547-556.

[46] C. Ratanamahatana, E. Keogh, Making time-series classification more accurate using learned constraints, in: Proceedings of SIAM International Conference on Data Mining, 2004, pp. 11-22.

[47] H. Shatkay, S. Zdonik, Approximate queries and representations for large data sequences, in: Proceedings of International Conference on Data Engineering, 1996, pp. 536-545.

[48] D. Srisai, C. Ratanamahatana, Efficient time series classification under template matching using time warping alignment, in: Proceedings of International Conference on Computer Sciences and Convergence Information Technology, 2009, pp. 685-690.

[49] U. Vespier, A. Knobbe, S. Nijssen, J. Vanschoren, MDL-based analysis of time series at multiple time-scales, in: Proceedings of European Conference on Machine Learning and Principles and Practice of Knowledge Discovery in Databases, 2012, pp. 371-386.

[50] U. Vespier, A. Knobbe, J. Vanschoren, S. Miao, A. Koopman, B. Obladen, C. Bosma, Traffic events modeling for structural health monitoring, in: Proceedings of Intelligent Data Analysis, 2011, pp. 376-387.

[51] U. Vespier, S. Nijssen, A. Knobbe, Mining characteristic multi-scale motifs in sensor-based time series, in: Proceedings of International Conference on Information and Knowledge Management, 2013, pp. 2393-2398.

[52] M. Vlachos, D. Gunopoulos, G. Kollios, Discovering similar multidimensional trajectories, in: Proceedings of International Conference on Data Engineering, 2002, pp. 673-684.

[53] G. Walraven, Basic Arrhythmias, Prentice Hall, 2010

[54] Q. Wang, V. Megalooikonomou, A dimensionality reduction technique for efficient time series similarity analysis, Inform. Syst. 33 (2008) 115-132.

[55] X. Wang, K. Smith, R. Hyndman, Characteristic-based clustering for time series data, Data Min. Knowl. Discov. 13 (2006) 335 -364.

[56] L. Wei, E. Keogh, H. Van Herle, A. Mafra-Neto, Atomic wedgie: Efficient query filtering for streaming times series, in: Proceedings of International Conference on Data Mining, 2005, pp. 490-497.

[57] L. Ye, E. Keogh, Time series shapelets: A new primitive for data mining, in: Proceedings of International Conference on Knowledge Discovery and Data Mining, 2009, pp. 947-956.

[58] B. Yi, C. Faloutsos, Fast time sequence indexing for arbitrary Lp norms, in: Proceedings of International Conference on Very Large Data Bases, 2000, pp. 385-394. 